\title{
Eduard Arzt awarded the highest European research prize
}

The materials scientist Professor Eduard Arzt, Scientific Director and Chairman of the INM - Leibniz Institute for New Materials, has been awarded an advanced grant of around $€ 2.5$ million by the European Research Council (ERC). Over the next five years, he intends to use this to develop three-dimensional structures and surfaces whose functions can be switched on and off via external stimuli. The advanced grants of the ERC honour outstanding achievements of European scientists in pioneering research.

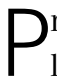
rofessor Arzt is pursuing various long-term goals with his research. On the one hand, for example, highly sensitive wafers or lens systems could eventually be picked and placed in production processes via switchable adhesion without leaving residues or causing damage and without using grippers or suckers. Medical implants, the adhesion of which does not commence until required, can make the work of surgeons easier. On the other hand, the switching of the new surface microstructures also provides the opportunity not only to affect adhesion but to selectively affect the sense of touch as well. For example, car steering wheels could in future send out a hazard warning through a sudden change in the surface; keyboard simulations on smooth touchscreens could be made to feel more real at the push of a button, thus making use of the sense of touch in communication between man and computer.

Whilst static adhesion systems for level hard surfaces have been subject to in-depth investigation for some time now, virtually no research has been con-

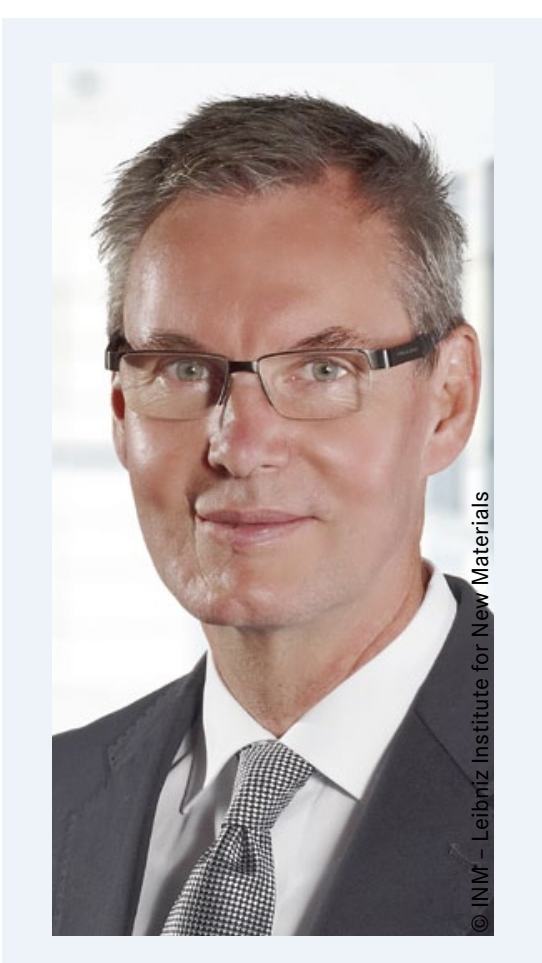

Prof. Eduard Arzt is conducting research into the targeted switching on and off of adhesion on soft surfaces etc. ducted on the simulation of switchable adhesion or adhesion on soft surfaces. "Controlled adhesion and detachment - this is a well-known principle in nature. Geckos use it to move on smooth, rough, flexible or rigid substrates, on walls or even upside down. Their adhesive ability is based on very fine hairs on their feet known as fibrils," explains the Scientific Director and Chairman at the INM. "With the ERC project, we are conducting research into a new generation of synthetic gecko structures. By changing the temperature or using electric fields or other external influences, it should be possible to switch adhesion on and off as required.

A second focus is on understanding how gecko structures can adhere to flexible surfaces - for example human skin. This principle is to be further developed for touch structures and biomedical applications. Ultimately, our aim is to demonstrate the feasibility of bio-inspired surfaces on a larger scale in order to provide a basis for cost-effective production," Arzt explains.

\section{Synthomer increases production capacity in Saudi Arabia}

\section{Synthomer Middle East is to ex- $\checkmark$ pand its production plant for \\ of the expansion is planned for mid- 2014.} acrylic and vinyl acetate dispersions in Dammam, Saudi-Arabia, with the aim of increasing the annual plant capacity to over 100,000 tonnes. With this expansion, the company intends to strengthen its position as a supplier of binding agents for coatings, construction chemicals, adhesives, textiles and floor coverings. Completion
"The market in the Middle East has continued to develop strongly in recent years, and this latest expansion to our Dammam facility is essential to ensure that we keep pace with the growing demands from our construction and coating customers in the region as well as providing us with the opportunity to introduce further cutting-edge technologies and develop our business in a broader range of market segments," said Synthomer Middle East's Managing Director, Shaz Rashid.

Synthomer Middle East is a jointventure between a subsidiary of Synthomer plc (United Kingdom) and Dhahran Chemical Industries and Marketing Company. 